\title{
Texture Detect on Rotary-Veneer Surface Based on Semi-Fuzzy Clustering Algorithm
}

\author{
Wei Cheng ${ }^{*}$, Ping Liang, and Suqun Cao \\ Faculty of Mechanical Engineering, Huaiyin Institute of Technology, \\ Huaian, Jiangsu Province, P.R. China 223003, \\ Tel.: +86-517-83559196 \\ hychwasina.com
}

\begin{abstract}
The texture of rotary-veneer can interference in defects detection, this paper presented a modified semi-fuzzy clustering (SFC) algorithm. SFC algorithm incorporates Fisher discrimination method with fuzzy theory using fuzzy scatter matrix. By iteratively optimizing the fuzzy Fisher criterion function, the final clustering results are obtained. SFC algorithm exhibits its robustness and capability to obtain well separable clustering results. This algorithm can detect the texture and defects on rotary-veneer surface exactly.
\end{abstract}

Keywords: Rotary-veneer, Semi-fuzzy clustering (SFC), Defects detection, Texture.

\section{Introduction}

Plywood grade was decided by the surface quality of rotary-veneer. The defect of rotary-veneer surface affects the quality of the wood-board panels products such as plywood, the computer vision system that forms using linear CCD gathers the image of veneer tape can be used automatic control and optimization of veneer tape cut out offer tech support. A goal of the rotary-veneer defects detection is that presents according to the defects different with the veneer background characteristic, and separates it from the veneer background. The superficial backgrounds of rotary-veneer include mainly each kind of shape and the relative order texture pattern. Due to textures and the most detects of veneer surface are the nature formations, their quality are the same, and their characteristic are similar. Rotary-veneer surface texture regular session disturbance defects characteristic extraction. Therefore, the research of the rotary-veneer surface texture detection may enhance the rotary-veneer detection precision, realizes automatic grading based on the computer vision veneer.

In reference (Ping Liang, Wei Cheng 2008), the texture of rotary-veneer can interference in defect detects, this paper presented a modified Fuzzy C-Mean algorithm (FCM). The approach of this algorithm is sample density of inter-class and distances of intra-class as comprehensive parameters, thereby to obtain the validity initial cluster centers. This algorithm can detect the texture and defects on rotary-veneer surface. Most of these methods like FCM are essentially rooted at the within-cluster scatter

\footnotetext{
* Corresponding author.
} 
matrix as a compactness measure, which means that the assumption that the clusters are hyperspheroidal is taken. In fact, real data sets seldom accommodate such an assumption. Except for the compactness measure, the concept of the separation measure such as the between-cluster scatter matrix should also be involved in the design of clustering methods such that well separated cluster can be obtained.

In this paper, we extend Fisher linear discriminant to its fuzzified version and define the fuzzy Fisher criterion function. Then a novel semi-fuzzy clustering (SFC) method is presented. Compared with FCM, the proposed algorithm has the following characteristics: (1) It directly uses the fuzzy FLD as its objective function, therefore, it is an unsupervised fuzzy partition clustering method. Its objective function integrates the fuzzy between-cluster scatter matrix well with the fuzzy within-cluster scatter matrix. (2) It incorporates the discriminating vector into its update equations such that the obtained update equations do not take commonly-used FCM-like forms. (3) It is more robust to noise and outliers than FCM.

\section{Fisher Criterion and Fisher Linear Discriminant}

Given c pattern classes $X^{(i)}=\left[x_{i}^{1}, x_{i}^{2}, \ldots, x_{i}^{N_{i}}\right]$ in the pattern set which contains $\mathrm{N}$ d-dimensional patterns, where $i=1,2, \ldots c, N_{i}$ is the number of all the patterns in the ith class, thus $N=N_{1}+N_{2}+\cdots+N_{c}$. The between-class scatter matrix $S_{b}$ and the within-class scatter matrix $S_{w}$ are determined by the following formulae:

$$
\begin{gathered}
S_{b}=\sum_{i=1}^{c} \frac{N_{i}}{N}\left(m_{i}-\bar{x}\right)\left(m_{i}-\bar{x}\right)^{T} \\
S_{w}=\frac{1}{N} \sum_{i=1}^{c} \sum_{j=1}^{N_{I}}\left(x_{j}^{i}-m_{i}\right)\left(x_{j}^{i}-m_{i}\right)^{T}
\end{gathered}
$$

Where $m_{i}$ denotes the mean of the ith class, $\bar{x}$ denotes the mean of all the patterns in the pattern set.

According to the scatter matrices, the Fisher criterion function can be defined as follows:

$$
J_{F C}(\omega)=\frac{\omega^{T} S_{b} \omega}{\omega^{T} S_{w} \omega}
$$

Where $\omega$ is an arbitrary vector in d-dimensional space. The Fisher optimal discriminate vector is $\omega^{*}$ corresponding to maximum of $J_{F C}(\omega)$, which is the eigenvector corresponding to maximum eigenvalue of the following eigensystem equation:

$$
S_{b} \omega^{*}=\lambda S_{w} \omega^{*}
$$

Where $\lambda$ is diagonal and consists of the corresponding eigenvalues. 


\section{Fuzzy Fisher Criterion and the Proposed Algorithm}

First, let us fuzzily the concept of the above Fisher linear discriminate (FLD).

Suppose that the membership function $u_{i j} \in[0,1]$ with $\sum_{i=1}^{c} u_{i j}=1$ for all $j$ and the index $m>1$ is a given real value, where $u_{i j}$ denotes the degree of the $j t h \mathrm{~d}$ dimensional pattern belonging to the ith class, we can define the following fuzzy within-class scatter matrix $S_{f w}$ :

$$
S_{f w}=\sum_{i=1}^{c} \sum_{j=1}^{N_{I}} u_{i j}^{m}\left(x_{j}-m_{i}\right)\left(x_{j}-m_{i}\right)^{T}
$$

And the following fuzzy between-class scatter matrix $S_{f b}$ :

$$
S_{f b}=\sum_{i=1}^{c} \sum_{j=1}^{N_{I}} u_{i j}^{m}\left(m_{i}-\bar{x}\right)\left(m_{i}-\bar{x}\right)^{T}
$$

Thus, we can derive a novel fuzzy Fisher criterion called fuzzy FLD as follows:

$$
J_{F F C}(\omega)=\frac{\omega^{T} S_{f b} \omega}{\omega^{T} S_{f w} \omega}
$$

In terms of the fuzzy FLD as above, we will derive a novel semi-fuzzy clustering algorithm based on fuzzy Fisher criterion. Maximizing $J_{F F C}$ directly in Eq.(7) is not a trivial mathematical derivation task due to the existence of its denominator. However, we can reasonably relax this problem by applying the following Lagrange multipliers $\lambda$ and $\beta_{j}(j=1,2, \cdots, n)$ together with the constraint $\sum_{i=1}^{c} u_{i j}=1$ to formula (7):

$$
F=\omega^{T} S_{f b} \omega-\lambda \omega^{T} S_{f w} \omega+\sum_{j=1}^{N} \beta_{j}\left(\sum_{i=1}^{c} u_{i j}-1\right)
$$

Setting $\frac{\partial F}{\partial \omega}, \frac{\partial F}{\partial m_{i}}, \frac{\partial F}{\partial u_{i j}}$ to be zero, we respectively have

$$
S_{f b} \omega=\lambda S_{f w} \omega
$$

Where $\lambda$ may be taken as the largest eigenvalue. 


$$
\begin{gathered}
m_{i}=\frac{\sum_{j=1}^{N} u_{i j}^{m}\left(x_{j}-\frac{\bar{x}}{\lambda}\right)}{\sum_{j=1}^{N} u_{i j}^{m}\left(1-\frac{1}{\lambda}\right)} \\
u_{i j}=\frac{F_{1}}{F_{2}}
\end{gathered}
$$

Where

$$
\begin{aligned}
& F_{1}=\left(\omega^{T}\left(x_{j}-m_{i}\right)\left(x_{j}-m_{i}\right)^{T} \omega-\frac{1}{\lambda} \omega^{T}\left(m_{i}-\bar{x}\right)\left(m_{i}-\bar{x}\right)^{T} \omega\right)^{\frac{1}{m-1}} \\
& F_{2}=\sum_{k=1}^{c}\left(\omega^{T}\left(x_{j}-m_{k}\right)\left(x_{j}-m_{k}\right)^{T} \omega-\frac{1}{\lambda} \omega^{T}\left(m_{k}-\bar{x}\right)\left(m_{k}-\bar{x}\right)^{T} \omega\right)^{\frac{1}{m-1}}
\end{aligned}
$$

When Eq.(11) is used, as stated in the above, $u_{i j}$ should satisfy $u_{i j} \in[0,1]$, hence, in order to satisfy this constraint, we let

$$
\begin{aligned}
u_{i j}= & 1 \text { and } u_{i j}=0 \text { for all } i^{\prime} \neq i, \text { if } \\
& \omega^{T}\left(x_{j}-m_{i}\right)\left(x_{j}-m_{i}\right)^{T} \omega \leq \frac{1}{\lambda} \omega^{T}\left(m_{i}-\bar{x}\right)\left(m_{i}-\bar{x}\right)^{T} \omega
\end{aligned}
$$

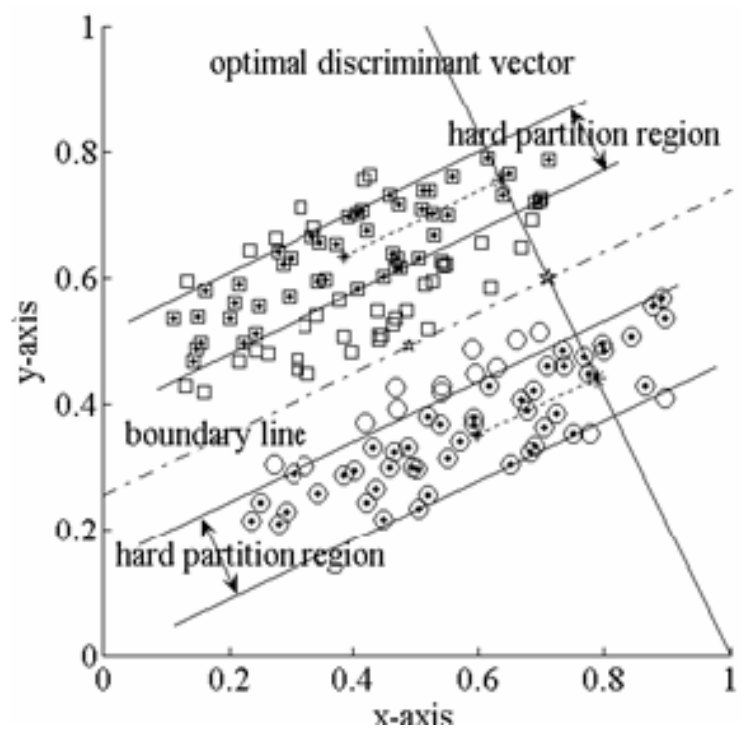

Fig. 1. FFC-SFC hard partition regions 
That is to say, if Eq.(12) holds, we take a hard partition for the pattern. The rational can be intuitively explained from a geometric viewpoint as shown in Fig. 1. In Fig. 1, all the patterns with one class $\square$ and the other class $\circ$, the two clusters center with * and the total mean point (i.e. the average point of all samples) with is are projected along dotted lines onto the optimal discriminating vector. Obviously, if the Euclidean distance between the projection of a pattern and the projection of certain cluster is equal to or less than multiplied by the Euclidean distance between the projection of this cluster and $\bar{t}$, then we should take a hard partition for this pattern.

From the above analysis, we can obtain a novel semi-fuzzy algorithm based on fuzzy fisher criterion (FFC-SFC).

\section{Algorithm FFC-SFC}

Step1. Set the given threshold $\mathcal{E}$, initialize $U=\left[u_{i j}\right]_{c \times N}$ and $m=\left(m_{1}, m_{2}, \cdots, m_{c}\right)$ using K-mean;

step2. Compute $S_{f w}, S_{f b}$ using Eq.(5), Eq.(6) respectively; Eq.(9);

step3. Compute the largest eigenvalue $\lambda$ and the corresponding $\omega$ using

Step4. Update $m_{i}$ and $u_{i j}$ using Eq.(10), Eq.(11) and Eq.(12) respectively;

Step5. Compute $J_{F F C}$ using Eq.(7);

Step6. If $J_{F F C}<\mathcal{E}$ or the number of iteration $\geq$ the given value, output the clustering result and then terminate, otherwise back to Step 2.

\section{The Analyses of Rotary-Veneer Surface Texture Detection}

In the research of rotary-veneer surface texture detection, a goal is to enhance the detection precision of rotary-veneer surface defects. Due to the textures show large expanse, continuously long linearity distribution. The textures occupy the big region in the rotary-veneer surface. But the defects of rotary-veneer surface occupy the small region, and they are regular, such as the shape of knots is an ellipse. Compares with the texture color, the defects color is also deep. Therefore, when we segment the rotary-veneer defect image, shape and color was chosen for this segmentation characteristic.

In fig.1, (a) image show rotary-veneer image; (b) image show used the FCM algorithm which this reference (Ping Liang, Wei Cheng 2008) proposed to segment this texture image; (c) image show used SFC to segment this texture image.

We can see that SFC has better segmentation accuracy, and is comparatively competitive to FCM. 


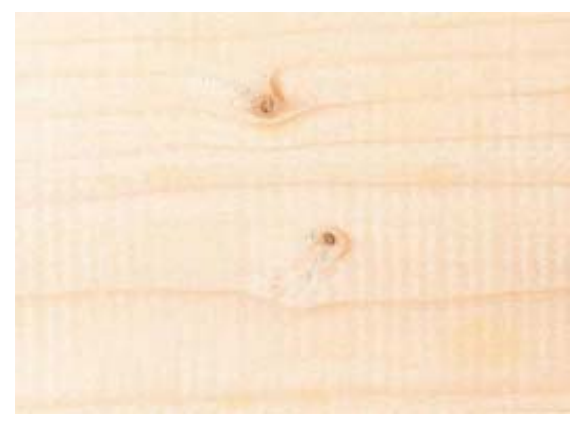

a) original image
b) $F C M$
c) $S F C$

Fig. 2. Rotary-veneer image detection result

\section{Acknowledgements}

Funding for this natural science research was provided by Jiangsu Provincial Department of Education (P.R. China) (No. 2005KJD520033).

\section{References}

Cao, S., Wang, S., et al.: Fuzzy Fisher Criterion Based Semi-Fuzzy Clustering Algorithm. Journal of Electronics \& Information Technology 30(9), 2132-2165 (2008) (in Chinese)

Liang, P., Cheng, W.: Texture Detection on the Rotary-Veneer Surface Based on FCM. Techniques of Automation and Applications (11), 79-85 (2008) (in Chinese) 